\title{
Janus Cornarius éditeur et commentateur du traité de Galien \\ Sur la composition des médicaments selon les lieux
}

\author{
ALESSIA GUARDASOLE
}

CNRS Paris

The Saxon humanist Janus Cornarius (ca. 1500-1558) devoted himself to edit, translate, and comment the extant works of Galen of Pergamon (129-ca. 216). In this study I deal with the Latin translation and commentary of Galen's work On the composition of drugs by site, edited by Cornarius (Basel, 1537). Namely, by investigating some passages of Galen's treatise, my effort is to throw light on Cornarius' working method, defining which literary and manuscript sources he used to improve and interpret thoroughly Galen's text.

T grand travail philologique qui fit suite à l'édition princeps des œuvres de Galien chez les Aldes en 1525 s'enrichit considérablement avec l'activité extrêmement féconde de Janus Cornarius ${ }^{1}$.

Après avoir acquis en 1535 l'édition Aldine de Galien, Cornarius consacra son travail d'interprétation aux traités galéniques qui n’avaient pas encore suscité l'intérêt des autres traducteurs, en choisissant premièrement les œuvres sur la respiration en raison de son importance physiologique (rem sine qua vivere non possumus), et deuxièmement sur la procréation ${ }^{2}$ en raison vraisemblablement des liens doctrinaux que l'on pouvait trouver avec les œuvres d'Hippocrate au sujet de la procréation et des affections de la femme.

Notre étude sera consacrée au travail du médecin allemand sur les textes galéniques qui a immédiatement suivi, à savoir l'édition de prestige dédiée à Albert de Mayence qui contient la traduction des dix livres du traité Sur la composition des médicaments selon les lieux ${ }^{3}$ (que j'appellerai désormais Secundum locos), publiée à Bâle chez Froben en 1537 et enrichie de façon tout à fait exceptionnelle par un commentaire continu de l'ouvrage de pharmacologie ${ }^{4}$, dans lequel Cornarius déploie tout son savoir philologique et médical. Comme 
on le déduit de la date de la préface au commentaire, Cornarius y avait travaillé déjà à partir de 1535 , donc antérieurement à la publication de sa première édition galénique (Bâle, 1536).

La traduction est réalisée à partir du texte de l'Aldine, seul témoin grec consulté (comme Cornarius le dit à plusieurs reprises dans son ouvrage) $)^{5}$; le texte galénique est corrigé tantôt ope ingenii, tantôt avec l'aide de l'ancienne traduction latine de Niccolò de Reggio ${ }^{6}$ (vetus interpres; cf. p. $357^{* 7}$ ad sufficiens itaque...] ...veterem Interpretem, Nicolaum Calabrum, aut quisquis is fuit...) et des passages parallèles des auteurs anciens grecs et latins ${ }^{8}$. La traduction est accompagnée d'un commentaire de Cornarius, le seul existant de ce traité galénique, et qui d’après son auteur était absolument nécessaire de par la nature même de l'œuvre, pharmacopée certes datée, mais d'intérêt encore bien actuel pour les médecins du XVI e siècle?.

L'intérêt premier de Cornarius dans son commentaire est de justifier ou d'appuyer une modification du texte ou une traduction personnelle qui s'écarte de la tradition ${ }^{10}$; il y a toutefois de longues notes consacrées aux médicaments ou aux simples les plus renommés, exotiques ou énigmatiques rencontrés dans le recueil pharmaceutique.

En raison de l'ampleur du traité de Galien et, par conséquent, du commentaire que Cornarius lui consacre, je me propose de présenter des cas emblématiques des notes du savant allemand pour en montrer la méthode de travail, les sources et les textes de référence.

Je commencerai par deux cas où Cornarius recourt, pour justifier sa position, à la traduction latine ancienne et aux textes parallèles d'autres médecins.

1)

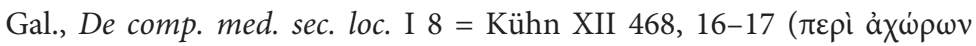
'Apхıүévouৎ):

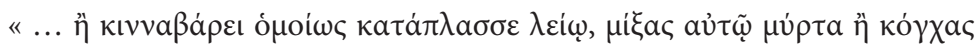

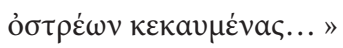

Cornarius (p. 25) : « aut cinnabari similiter tritum imponito. licebit et myrti baccas, aut ordeum ustum, aut argenti spumam, aut cerussam, aut ostreorum testas ustas ammiscere ». 
Il s'agit d'un remède contre la teigne (ả $\chi \omega \dot{\omega} \rho)^{11}$, tiré de l'œuvre d'Archigène. Le texte édité dans Kühn était déjà celui de l'Aldine (1525, II 95v',4), de l'édition de Bâle $(1538$, II 166, 49) et du texte grec de l'édition de René Chartier (1639, XIII $348 \mathrm{E})^{12}$.

Dans la note consacrée à ce passage, Cornarius (p. 411), après avoir donné la leçon de l'Aldine (exemplar grecum Venetum), fait appel à la traduction de Niccolò (Bonardus, I 384, $2^{13}$; Surianus, fol. 324, $2^{14}$ ) : « vetus autem Interpres

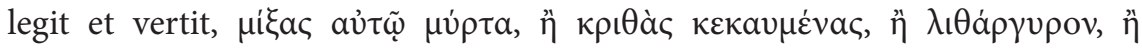

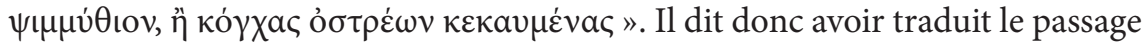
en fonction de la traduction latine, ainsi que de la suite du passage galénique (« atque hoc non tam ex veteris Interpretis praescripto faciendum est, quam ex ipsius Galeni declaratione, quae paulo post sequitur... »). En effet, les trois

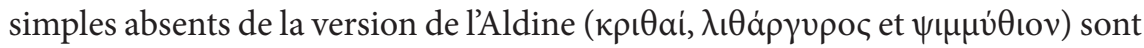
repris dans la discussion que Galien fait des ingrédients utilisés par Archigène contre la teigne (respectivement Kühn XII 471, 10. 472, 16. 473, 5).

Dans ce cas, donc, Cornarius s'appuie, pour améliorer le texte galénique, sur l'ancienne traduction latine, confirmée par Galien lui-même - d'après la 'règle d'or' propre déjà à la philologie alexandrine de « rendre clair [Galien] par [Galien] $»^{15}$.

Aurait-il eu accès aux sources manuscrites, il aurait pu constater que deux témoins du Secundum locos, les manuscrits Vaticanus Reginensis gr. 172 (XIV ${ }^{\mathrm{e}}$ siècle) ${ }^{16}$ et Laurentianus Pl. LXXV 8 (XV siècle) ${ }^{17}$, transmettent (respectivement

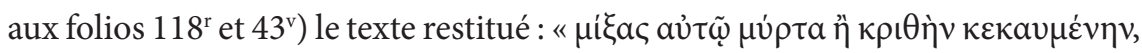

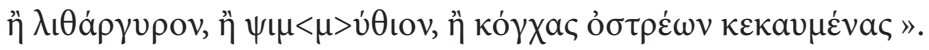

2)

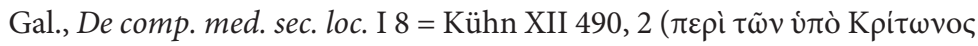

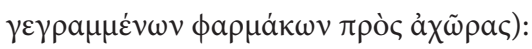

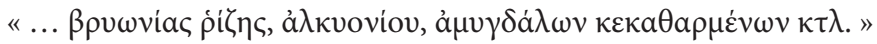

Cornarius (p. 31) : " radicis bryoniae, alcyonii, amygdalarum repurgatarum...».

Dans cette partie de recette de $\sigma \mu \tilde{\eta} \gamma \mu \alpha$ (une sorte de détergent sous forme d'onguent) contre la teigne, tirée de l'œuvre de Criton, on peut identifier de la part de Cornarius la même démarche analytique que dans le passage cité il y a 
peu : le texte de l'Aldine posant un problème d'ingrédients, l'érudit recourt à la fois à la traduction latine et à Galien lui-même dans la suite de ce passage.

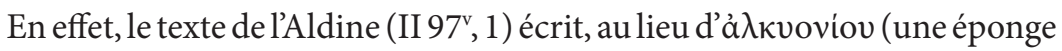

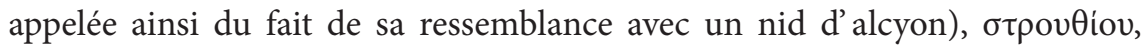
" la saponaire ", avec un astérisque superposé (ce qui signale dans l'édition de 1525 une leçon fort probablement corrompue). Dans son commentaire (p. 415),

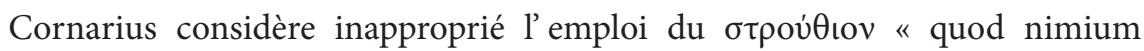
calfaciat et resiccat » et préfère lire ả $\lambda$ kvovíov, en s'appuyant sur la présence de cet ingrédient dans les recettes de deux autres $\sigma \mu \eta \dot{\gamma \mu \alpha \tau \alpha}$ transmises peu après (Kühn XII 491, 6. 492, 4), sur le témoignage de Dioscoride (De mat. med. V 118 = Wellmann III 87, 7-88, 13), ainsi que sur celui de Niccolò de Reggio qui traduit (Bonardus, p. 386, 2 ; Surianus, p. 326, 2) spume maris, " quomodo alias semper alcyonium vertere solet ${ }^{18}$.

Dans les exemples qui suivent, l'érudit allemand fait appel à son ingenium pour modifier le texte transmis.

1)

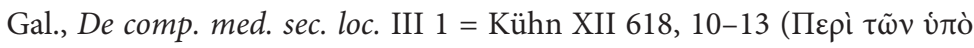

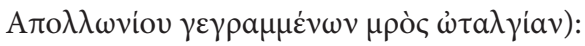

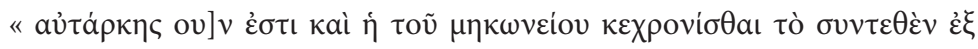

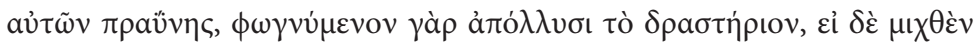

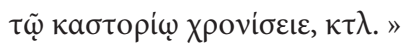

Cornarius (p. 65) : « sufficiens itaque est etiam papaveris succi facultas, si ex eo quod eorum compositio diu ante praeparata, et iam inveterata sit, ipsius vires leniantur, torrefactis enim efficaciam perdit. Si vero castorio mixtus ad tempus aliquod inveterascet...».

Il s'agit d'un passage problématique, perturbé dans la tradition manuscrite, tiré d'un chapitre d'Apollonios sur les remèdes contre les douleurs d'oreille. Galien y commente négativement la pertinence de torréfier le suc de pavot et le castoréum, ce qui était préconisé par Apollonios, ainsi que par d’autres médecins de grande renommée.

La note consacrée à ce passage dans le commentaire (p. $357^{\star 19}$ ) s'ouvre avec la constatation que la lecture de l'Aldine (II 110, 1-3) est manifestement

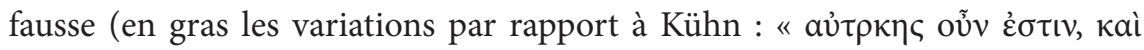




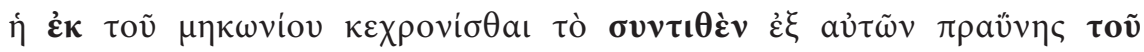

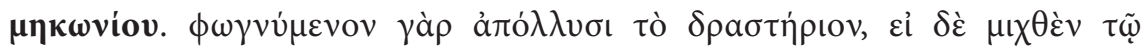
$\delta \rho \alpha \sigma \tau \eta \sigma i(\omega) \chi \rho o v i \sigma \varepsilon \varepsilon \varepsilon, \kappa \tau \lambda$.) », d'où la nécessité d'intervenir sur le texte pour le rendre compréhensible. Il propose donc de lire, dans la première partie

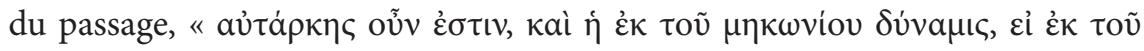

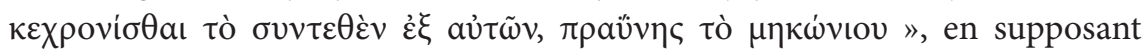

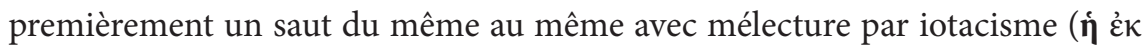

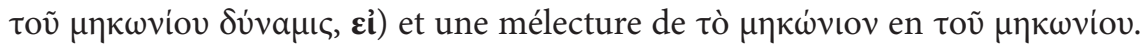
La deuxième partie non plus n' est pas exempte d'erreurs, vu que Cornarius

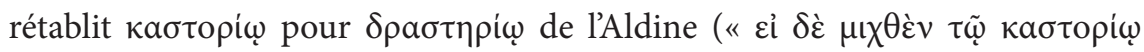

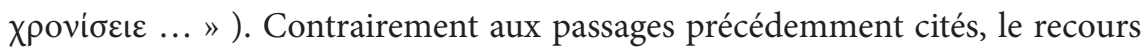
à l'ancienne traduction latine prouve seulement que le traducteur lisait déjà un texte corrompu dans son modèle ("Nicolaum Calabrum, aut quisquis is fuit, hunc locum non integrum habuisse »), qu'il a essayé de traduire, pour sa première partie, de façon compréhensible, mais sans respecter la lettre du passage (" quae lectio rectam sententiam refert, sed tamen aliis verbis quam exemplar graecum vulgatum ostendit») : cf. Niccolò (Bonardus, fol. 401, 1 ; Surianus, fol. 340, 2) sufficit igitur ut mitigetur opium ex diuturna compositione

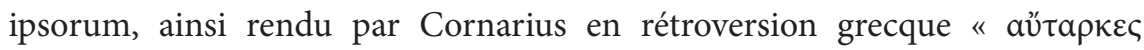

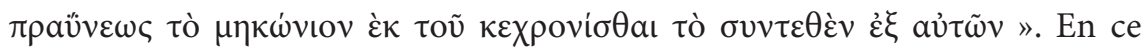
qui concerne la deuxième partie, il traduit le texte corrompu, qui est propre

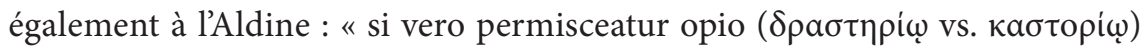

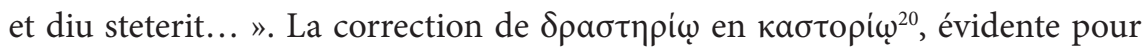
l'ingenium de Cornarius à partir du contexte, est confirmée dans toute la tradition manuscrite du traité, à l' exception du Vaticanus Palatinus gr. 54 $\left(\mathrm{XV}^{\mathrm{e}}-\mathrm{XVI}^{\mathrm{e}}\right.$ siècles$)$, modèle du manuscrit fragmentaire Rosanbo $286\left(\mathrm{XVI}^{\mathrm{e}}\right.$ siècle), copie d'impression de l'Aldine pour le Secundum locos, dont les folios relatifs à cette partie du traité galénique ne nous sont pas parvenus ${ }^{21}$.

2)

Gal., De comp. med. sec. loc. I 6 = Kühn XII 460, 13:

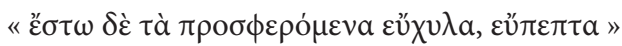

Cornarius (p. 23) : « sint autem cibi quibus vescuntur boni succi, faciles concoctu». 
Il s'agit de préceptes tirés d'Archigène d'Apamée, concernant le régime des patients souffrant de pellicules ( $\pi ı \tau v p i a \sigma ı \varsigma^{22}$ ) : l'Aldine (II 94v , 28) donne -

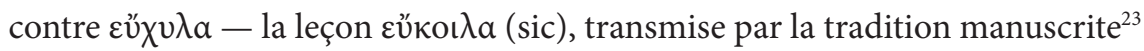
et reprise dans l'édition de Bâle (II 165, 32). Dans son commentaire, Cornarius (p. 407) enregistre la leçon fautive de l'Aldine et donne la variante de l'ancienne

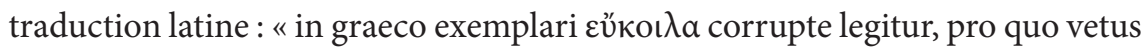

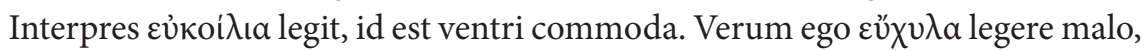
hoc est boni succi » (aliments succulents $v s$. aliments qui relâchent le ventre). En effet, Niccolò traduit (Bonardus, p. 383, 2 ; Surianus, p. 323, 2) bonificantia ventrem, leçon qui est confirmée également par la tradition indirecte (cf. Aetius,

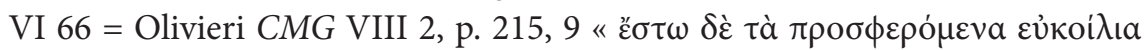
$\varepsilon \cup ̋ \pi \varepsilon \pi \tau \alpha »$ ); cependant c'est la correction $\varepsilon u ̈ \chi u \lambda \alpha$ de Cornarius qui est entrée dans les éditions grecques à partir de celle de Chartier.

À côté de son intérêt principal d’amender le texte grec, Cornarius consacre également tous ses efforts philologiques pour atteindre la traduction exacte de termes ambigus, n'hésitant pas à rectifier les interprétations que des illustres prédécesseurs en avaient données. Nous trouvons un très riche exemple de ces cas dans la note consacrée aux «Smegmata ad achoras, furfures, asperitates et scabies» (p. 414).

3)

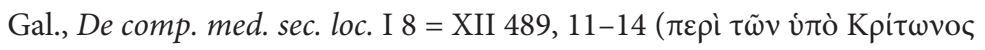

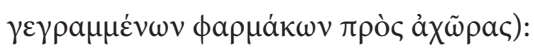

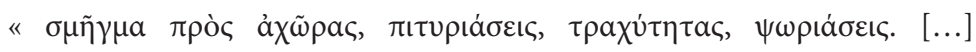

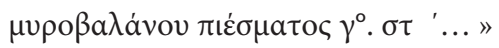

Cornarius (p. 31) : «smegma ad achoras, furfures, asperitates et scabies. [...] retrimentorum quae ab expressa myrobalano reliqua sunt sexuncem ...».

Cornarius consacre sa note du commentaire (p. 414 sq.) à la traduction

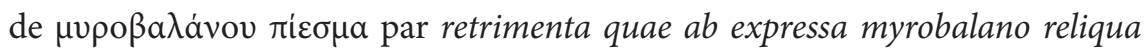
sunt, c' est-à-dire " résidus qui subsistent après l'expression du myrobalan ", plutôt que par "suc de myrobalan », comme le sens le plus répandu du mot

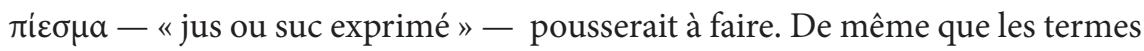

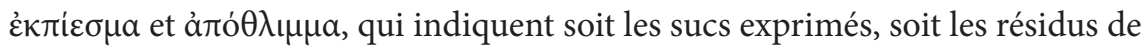


la pression, dans plusieurs passages le mot $\pi i \varepsilon \sigma \mu \alpha$ présente la même ambiguïté et requiert, pour une interprétation correcte, une analyse attentive du contexte.

Cornarius fait donc appel à une très riche série de passages parallèles pour prouver l'exactitude de sa traduction. Dans son « appel à témoins » défilent ainsi, à l'appui du sens de " résidu », Dioscoride, De mat. med. IV 157, 2 (Wellmann

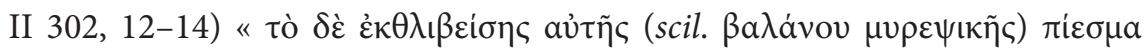

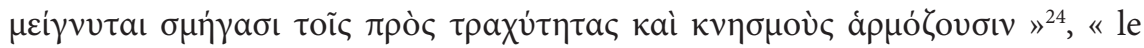
résidu de l'expression du gland d'Égypte est mélangé aux onguents détergents efficaces contre les rugosités et les démangeaisons "; Aetius, XIII 128 (éd. Cornarius, Lyon 1549, [col. 824]), «(Ad pustulas psydracas Graecis appellatas) aliud smegma. Cimoliae drach. II, farinae ervi dra. II, quod ex myrobalano contusa et expressa reliquum est tandundem ... »; Galien, De comp. med. sec. loc. II 1 (Kühn XII 558, 4 sq.), dans la recette de l'épithème de Cariclès contre

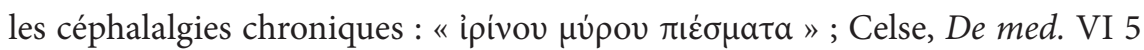
(De varis et lenticulis et ephelide et eorum curationibus), qui rend $\mu$ voßa $\beta$ ávov

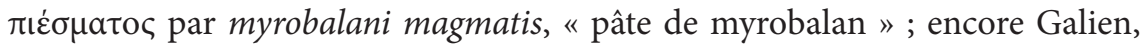

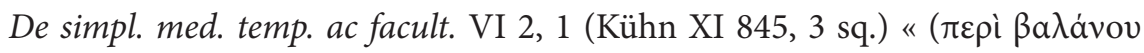

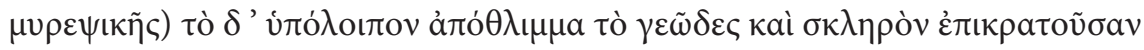

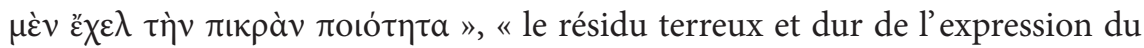
gland a la qualité âcre dominante » et De meth. med. XIII 15 (Kühn X 911, 4)

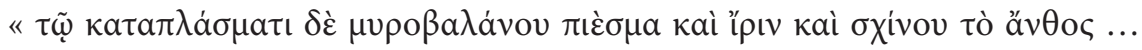
$\mu \tilde{\zeta}$ ov », " au cataplasme mêle du résidu de l'expression de myrobalan et de l'iris et de la fleur de lentisque».

C'est à ce moment de son argumentation que Cornarius engage une assez longue digression concernant l'interprétation erronée faite par l'illustre médecin et humaniste anglais Thomas Linacre (ca. 1460-1524) de ce passage dans sa traduction du De methodo medendi $i^{25}$ (fol. 167v) : « cataplasmati vero id quod ex unguento et palmulis fit, myrobalanu piesma vocant, et irim, et iunci florem ... miscebit ${ }^{26}$. Il y a dans la suite une tentative de la part de Cornarius d'expliquer l'erreur de l'illustre prédécesseur : Linacre aurait été induit en erreur par Celse, De med. IV 9 (= IV 16), où, à propos d'un emplâtre contre la lienosis - " maladie de la rate ", il est dit : "fit ex unguento et palmulis, quod $\mu$ poßá $\lambda$ avov Graeci vocant "; Cornarius propose donc (p. 415) de corriger ce passage de Celse en « fit ex unguentariis glandibus quas $\mu \nu \rho \circ \beta a \lambda a ́$ vovৎ vocant », en s'appuyant

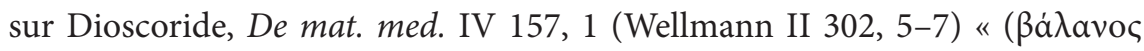

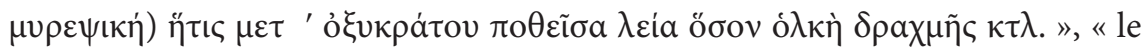


gland d'Égypte, si on le boit avec de l'oxycrat dans le poids d'une drachme, fond la rate. Il est également utilisé en cataplasme sur les mêmes patients avec de la farine d'ivraie... ». Une autre explication (pourtant jugée assez improbable par Cornarius) serait que Linacre ait suivi dans son interprétation le poète Martial, qui dit, dans ses Apophoreta 57, Myrobalanum. «Quod nec Vergilius nec carmine dicit Homerus, / Hoc ex unguento constat et ex balano ».

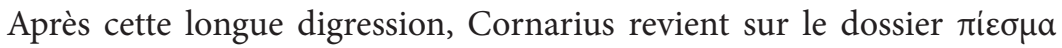
comme " résidu d'expression » et cite son dernier passage parallèle : Aetius,

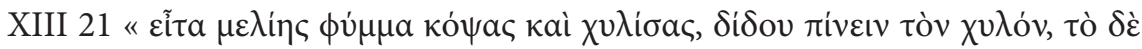

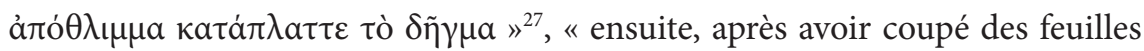
de frêne et après avoir extrait leur suc, donnes-en à boire ; quant au résidu, applique-le en cataplasme sur la morsure ».

Avec la même attention minutieuse, Cornarius passe maintenant à la citation de passages qui attestent le sens plus courant de « suc, jus " pour

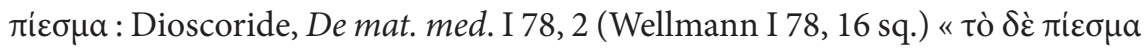

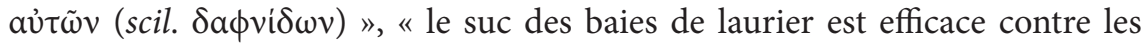
douleurs d'oreille, instillé avec du vin vieux et de l'huile de roses »; Galien, De comp. med. sec. loc. II 1 (Kühn XII 551, 16), dans un remède d'Archigène,

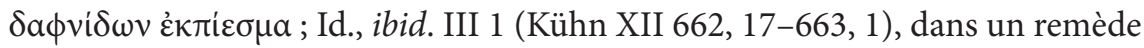
tiré de l'œuvre d'Apollonios contre les inflammations aux oreilles, " $\beta a \lambda a ́ v o v$

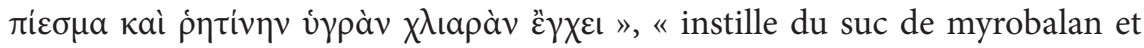
de la résine liquide tiède "; Id., ibid. III 1 (Kühn XII 620, 10 sq.), dans un

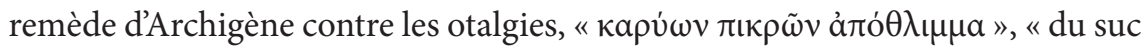
de noix amères $»^{28}$; Dioscoride, De mat. med. I 110, 2 (Wellmann I 104, 9 sq.)

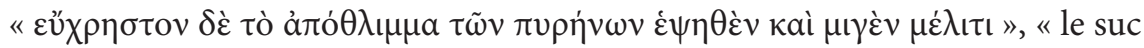
des pépins (scil. de la grenade) est très utile, cuit et mélangé avec du miel ». Si tous ces exemples prouvent bien, à partir du contexte, qu'il faut interpréter les

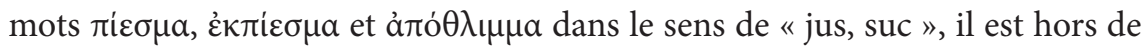
doute (« certum est ») que le même Dioscoride, quand il présente les propriétés

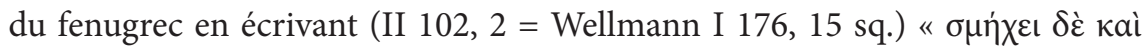

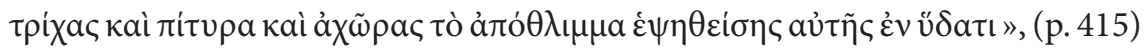

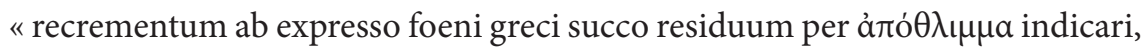
utpote quod crassum et terreum extersioni est aptum, neque curandum quid hic et in plerisque aliis locis Interpretes reddant ».

À l'aide de ces quelques exemples choisis, je crois avoir montré avec quelle érudition et acuité d'analyse Janus Cornarius intervient sur le texte du 
Secundum locos galénique, en réalisant non seulement une traduction attentive du texte grec, mais un vrai travail d'édition ; le commentaire continu qui l' accompagne - exemple plutôt rare pour un texte médical ancien - est une sorte d'invitation dans l'atelier de travail du philologue de la Renaissance et nul éditeur moderne du traité ne pourra l'ignorer, en réservant à son auteur une place méritée dans l'apparat critique.

\section{Notes}

1. Sur cet érudit allemand de Zwickau, dont le nom de naissance était Johann Haynpol, cf. principalement - avec les bibliographies citées - B. Mondrain, "Éditer et traduire les médecins grecs au XVIe siècle. L'exemple de Janus Cornarius », in D. Jacquart éd., Les voies de la science grecque. Études sur la transmission des textes de l'Antiquité au dix-neuvième siècle (Genève : Droz, 1997), p. 391-417, et M.-L. Monfort, L'apport de Janus Cornarius (ca. 1500-1558) à l'édition et à la traduction de la collection hippocratique (Thèse, Université de Paris IV-Sorbonne, 1998), notamment p. 9-81.

2. Cf. M.-L. Monfort, L'apport de Janus Cornarius, p. 68 sq. et p. 271 sq., nº 10 : Claudi Galeni medici praestantissimi libri V. jam primum in latinam linguam conversi, Jano Cornario medico interprete De causis respirationis, Liber I, De utilitate respirationis, Liber I, De difficultate respirationis, Libri III. ... De uteri dissectione, Liber I, De foetus formatione, Liber I, De semine, Libri II, Basileae, Froben, 1536.

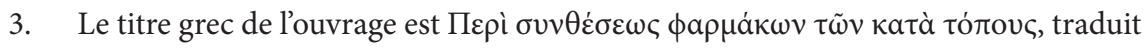
en latin en De compositione medicamentorum secundum locos.

4. Opus medicum practicum, varium, vere aureum, et postremae lectionis. Claudii Galeni Pergameni, medici longe omnium clarissimi De compositione pharmacorum localium, sive secundum locos, Libri decem, recens fideliter \& pure conversi a Iano Cornario medico physico. Iani Cornarii medici physici Commentariorum medicorum in eosdem Galeni libros conscriptorum Libri decem. In quibus omnes corrupti illorum librorum loci restituuntur, \& omnes difficiles exponuntur, ampliusque aliquot milia rerum ac locorum in graecis ac latinis medicis obiter explicantur, emendantur, ac restituuntur, Basileae, Froben-Episcopius, 1537 (consultable en ligne sur le site de la Bibliothèque Interuniversitaire de Médecine de Paris [http:// www.bium.univ-paris5.fr/histmed/medica/cote?00065x01]). 
5. Voir, par exemple, déjà dans la préface à la traduction, adressée à Albert de Mayence et datée de 1537 : « ... accedit ad hanc altera difficultatis causa, quod exemplar Venetiis invulgatum, quod in his vertendis unicum sequuti sumus, tam corruptum... "; p. 388 ad spumae nitri... «In graeco exemplari Veneto, quod solum in his vertendis in manum habuimus...».

6. Malgré la richesse des recours à l'ancienne traduction latine de la part de Cornarius, je n'ai pas pu repérer un passage péremptoire qui m’aide à comprendre s'il utilisait la traduction de Niccolò publiée dans l'édition de Diomedes Bonardus, imprimée à Venise en 1490, ou celle, considérablement retravaillée, publiée par Hieronymus Surianus à Rimini en 1502. Cf., sur les deux éditions, S. Fortuna, "Galeno latino, 1490-1533 », in Medicina nei secoli 17.2 (2005), p. 469-505 (notamment p. 472-476) ; sur l'utilisation de l'édition de Surianus, plutôt que de celle de Bonardus, par les érudits de la Renaissance (notamment par John Caius), A. Guardasole, «Les marginalia de John Caius au De compositione medicamentorum secundum locos de Galien dans l'édition de Bâle (1538) de l'Eton College ", in V. Boudon-Millot, A. Garzya, J. Jouanna et A. Roselli, éd., Storia della tradizione e edizione dei medici greci, Atti VI Coll. intern. (Paris, 12-14 avril 2008), (Naples : D’ Auria Editore, 2010, Collectanea 27) p. 344-346. Sur John Caius, les travaux de Vivian Nutton restent des ouvrages de référence : V. Nutton, « John Caius and the Linacre Tradition », Medical History 23 (1979), p. 373-391 ; Id., « John Caius and the Eton Galen: medical philology in the Renaissance ", Medizinhistorisches Journal 20 (1985), p. 227-52, réédité dans Id., From Democedes to Harvey: Studies in the History of Medicine (London: Variorum Reprints, 1988); Id., John Caius and the Manuscripts of Galen (Cambridge: The Cambridge Philological Society, 1987).

7. Je note avec un astérisque un problème dans la numérotation des pages du commentaire de Cornarius : la p. $357^{\star}$ est en réalité la p. 457 . Il y a en effet une perturbation dans la notation de la séquence des pages, qui comporte une notation de la page 418 comme 318 , des pages $427-428$ comme $327-328$, des pages $454-463$ comme 354-363 et 465-649 (fin du volume) comme 365-549.

8. Voir, dans la préface au commentaire, adressée à Philippe ${ }^{\text {er }}$, landgrave de Hesse, et datée de 1535 : «ad hanc itaque rem integre praestandam videbam mihi in primis opus esse emendatioribus exemplaribus, ex quorum collatione non desperabam auxilium vel certissumum nobis accessurum, et propterea collationis laborem, ut longe leviorem libentius omnino pertulissemus, quam quod alicubi omni exemplarium ope destituti, mera divinatione uti coacti fuimus [...] Maiorem confidentiam in optimis quibusque, tum graecis, tum latinis medicis autoribus 
locavimus, atque ex ipsorum collatione plerosque omnes locos et convertimus, et exposuimus, simul in integrum ex eorundem fide restitutos ».

9. Cf. Praef. ad comm. : « ita sane visum fuit nullum ex omnibus Galeni operibus maiore periculo corruptum haberi ac legi, quam hoc ipsum de pharmacorum compositione, praesertim si his pharmacis tuto ac feliciter uti velimus, quod ut ego mihi faciendum esse penitus decrevi, circa aegros qui se in posterum nostrae curae concredent...».

10. Nulle mention n' est faite, dans le commentaire, de la traduction du même traité Secundum locos, éditée à Paris en 1535 par Guinterius Andernacus (Johann Guenther von Andernach), Claudii Galeni Pergameni de compositione medicamentorum secundum locos, seu quae unicuique corporis parti conveniunt, libri decem, opus et eruditum et omnibus medicis necessarium, nunc primum latinitate donatum ac in lucem editum per Ioannem Guinterium Andernacum, Parisiis: Ex officina Simonis Colinaei, 1535. La date de 1535 apposée à la préface du commentaire cornarien (cf. supra) fait songer à un travail antérieur à l'édition de Guinterius.

11. Cf. F. Skoda, Médecine ancienne et métaphore. Le vocabulaire de l'anatomie et de la pathologie en grec ancien (Paris : Peeters, Selaf, 1988), p. 236.

12. La traduction latine éditée par l'éditeur vendômois prend en compte l'intégration de Cornarius (cf. infra), ce qui n'est pas surprenant : Chartier, en introduisant les concisae notae et variae lectiones à la fin du volume XIII (p. 1018-1020), dit avoir pris en considération pour l'édition du traité la traduction de Janus Cornarius (ainsi que celle de Guinterius Andernacus).

13. Vu l'absence de numérotation de folios/pages, je me réfère pour l'édition de Bonardus à la pagination de la numérisation de cet ouvrage disponible sur le site de la BIUM (http://www.bium.univ-paris5.fr/histmed/medica.htm).

14. Je me réfère à l'exemplaire, disponible en ligne, sur le site de la bibliothèque de l'Université de Göttingen (Göttinger DigitalisierungsZentrum [GDZ], http://gdz. sub.uni-goettingen.de).

15. En paraphrasant la célèbre devise de la philologie homérique d'époque alexandrine :

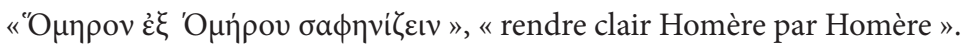

16. H. Stevenson, Codices manuscripti Graeci Reginae Suecorum et PII PP. II Bibliothecae Vaticanae (Romae : ex typographeo Vaticano, 1888), p. 116.

17. Æ.M. Bandinius, Catalogus codicum manuscriptorum Bibliothecae Mediceae Laurentianae, III, Florentiae 1770 (phot. 1961), p. 153. 


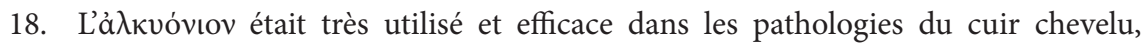
notamment les alopécies : cf. Gal., De comp. med. sec. loc. I 2 passim (Kühn XII 381-426 passim) ; De simpl. med. fac., VII 58 (Kühn XII 48, 17 sq.)

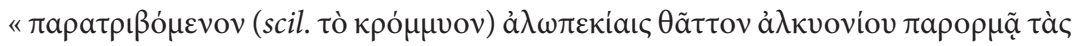
$\tau \rho i x a \varsigma$ », "frotté sur les parties atteintes d’alopécie, l’oignon fait pousser les cheveux plus rapidement que l'alcyonion ». En revanche, du $\sigma \tau \rho o u ́ \theta$ เov on employait surtout la racine pour ses propriétés nettoyantes et irritantes (Gal., De simpl. med. fac. VIII

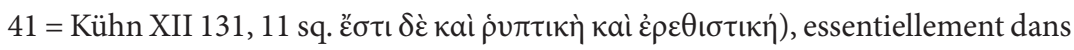
la préparation de sternutatoires et en gynécologie comme emménagogue et abortif (cf. déjà Hipp., Nat. mul. 32, 37 = Littré VII $354=$ Bourbon p. 33, 13). À côté de l'emploi topique de la saponaire dans un emplâtre cicatrisant d'Andromaque (Gal., De comp. med. per gen. II 15 = Kühn XIII 531, 7), j'ai trouvé l'attestation de son utilisation dans les cas de dermatoses dans Dioscoride, De mat. med. II 163, 1,

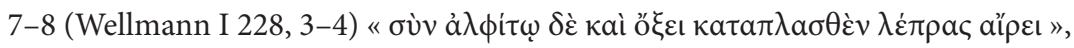
" appliqué sous forme de cataplasme avec de la farine et du vinaigre, il fait cesser les léprai ». S'agissant dans notre cas d'un remède contre la teigne, qui provoque une desquamation de l'épiderme tout comme les léprai, je trouve préférable de garder $\sigma \tau \rho o v \theta i o v$, qui est la leçon commune de tous les manuscrits ainsi que la

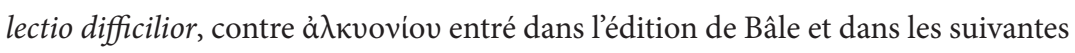
à partir de la traduction de Niccolò.

19. Cf. supra, note 6 .

20. Elle est intégrée déjà dans l'édition de Bâle, II 190, 33 et reprise dans celle de Chartier, XIII 396 E.

21. Cf. A. Guardasole, "Un nouveau modèle de l'Aldine de Galien : le manuscrit Rosanbo 286 ", in V. Boudon-Millot, A. Guardasole et C. Magdelaine éd., La science médicale antique : nouveaux regards, Mélanges offerts à M. Jacques Jouanna (Paris : Beauchesne, 2008) p. 235-247 (notamment p. 236, note 5, et 242-244).

22. Cf. F. Skoda, Médecine ancienne et métaphore, p. 235-238.

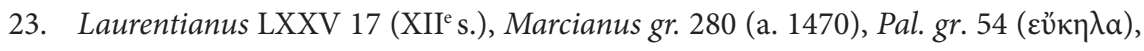
Parisinus gr. 2164 (XVI ${ }^{\mathrm{e}}$ s.), om. cett. codd.

24. Le texte cité par Cornarius, d'après l'édition princeps Aldine de 1499, diffère

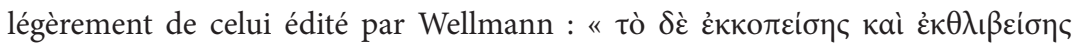
aủंñ

25. Galeni Methodus medendi, vel de morbis curandis Thoma Linacro Anglo interprete Libri quatuordecim (Parisiis: Didier Maheu, 1519). J'en ai consulté l'exemplaire conservé à Paris, à la Bibliothèque Interuniversitaire de médecine. 
26. Les critiques à Linacre pour des interprétations erronées du texte de Galien ne sont pas rares dans le commentaire de Cornarius : je citerai, en guise d'exemple, p. $380 \mathrm{Si}$ tamen...] « ... ceratum liquidum fit, quod nescio cur humidum potius,

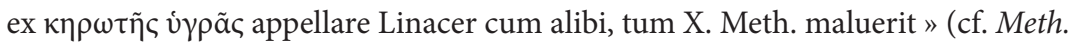
med. X 9 [Kühn X 703, 1] = Linacre, fol. 129v) ; p. 406 Faciunt enim Nigra...] « ... non de panibus in furno coctis accipiendum est, velut Linacer reddidit, sed de his qui sub Clibani textu coquuntur» (cf. Meth. med. VIII 2 [Kühn X 548, 12] = Linacre, fol. 101) ; p. 413 Mannae thuris tantundem] « ... sed lectio corrupta est in Graeco exemplari ... quae res fecit ut Interpres verterit "Componitur ex iride et aristolochia et ervo et thure et manna, i.e. cortice thuris" " (cf. Meth. med. III 2 [Kühn X 164, 6] = Linacre, fol. 30v); p. 419 Refrigerabis...] « ... Quo loco Interpres Linacer non intellexit, quae esset quam Romani decoctam appellare. $\delta\rceil \kappa o ́ \kappa \tau a v$

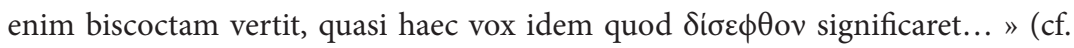
Meth. med. VII 4 [Kühn X 467, 17-468, 1] = Linacre, fol. 87).

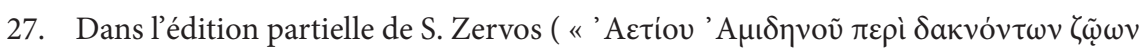
кaì loßó $\omega \omega v$ », Athena 18 [1906], p. 264-292), le passage se trouve au chapitre 23,

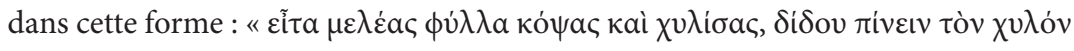

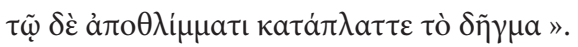

28. Les commentateurs anciens et modernes proposent l'équivalence entre les kápva

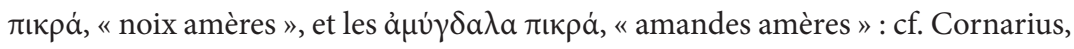
Opus medicum practicum, p. 387 («amaras nuces ... hic Archigenes dixit quas supra Crito et Cleopatra amygdalas amaras appellaverunt, id quod et Plinius et Celsus in usu habent ») ; Ivan Garofalo ad Anonym. Paris. 37, 12 (p. 197). 
